\title{
THE WIND OF ROTATING B SUPERGIANTS. I. DOMAINS OF SLOW AND FAST SOLUTION REGIMES
}

\author{
R. O. J. Venero ${ }^{1}$, M. Curé ${ }^{2}$, L. S. Cidale ${ }^{1,3}$, and I. Araya ${ }^{2}$

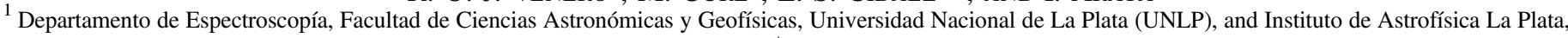 \\ CCT La Plata, CONICET-UNLP, Paseo del Bosque S/N, 1900 La Plata, Argentina; roberto@fcaglp.unlp.edu.ar \\ ${ }^{2}$ Instituto de Física y Astronomía, Facultad de Ciencias, Universidad de Valparaíso Av. Gran Bretaña 1111, Casilla 5030, Valparaíso, Chile \\ Received 2015 November 27; accepted 2016 March 1; published 2016 May 2
}

\begin{abstract}
In the scenario of rotating radiation-driven wind theory for massive stars, three types of stationary hydrodynamic solutions are currently known: the classical (fast) $m$-CAK solution, the $\Omega$-slow solution that arises for fast rotators, and the so-called $\delta$-slow solution if high values of the $\delta$ line-force parameter are allowed independently of the rotation speed. Compared to the fast solution, both "slow solutions" have lower terminal velocities. As the study of the parameter domain for the slow solution is still incomplete, we perform a comprehensive analysis of the distinctive flow regimes for B supergiants that emerge from a fine grid of rotation values, $\Omega$, and various ionization conditions in the wind $(\delta)$ parameter. The wind ionization defines two domains: one for fast outflowing winds and the other for slow expanding flows. Both domains are clear-cut by a gap, where a kink/plateau structure of the velocity law could exist for a finite interval of $\delta$. The location and width of the gap depend on $T_{\text {eff }}$ and $\Omega$. There is a smooth and continuous transition between the $\Omega$-slow and $\delta$-slow regimes, a single $\Omega \delta$-slow regime. We discuss different situations where the slow solutions can be found and the possibility of a switch between fast and slow solutions in B supergiant winds. We compare the theoretical terminal velocity with observations of B and A supergiants and find that the fast regime prevails mostly for early B supergiants while the slow wind regime matches better for A and B mid- and late-type supergiants.
\end{abstract}

Key words: hydrodynamics - stars: early-type - stars: mass-loss - stars: winds, outflows

\section{INTRODUCTION}

The standard theory of radiatively driven stellar winds, which is based on the works of Castor et al. (1975), Abbott (1982), Friend \& Abbott (1986), and Pauldrach et al. (1986), can be considered as a solid scenario to describe the wind structure of hot evolved stars. This theory includes the main features needed to successfully represent observed stellar wind conditions (mass loss and terminal velocity) of early B- and O-type supergiants. However, large discrepancies are still observed in the estimates of the wind parameters of supergiants of mid-B, late B, and A types (see, e.g., Verdugo et al. 1999; Crowther et al. 2006; Markova \& Puls 2008; Searle et al. 2008).

Standard models of radiation-driven winds generally make use of approximations that reduce considerably the complexity of the computation of the hydrodynamical solutions. One example is the extensive use of a simple parameterization for the velocity field, the so-called $\beta$-law, first proposed by Lamers \& Rogerson (1978). It is frequently used as an extension of a direct result from the $C A K$ theory (Castor et al. 1975), where pressure gradients are neglected. This approximation has been proved to be very effective and efficient to describe the winds of O- and early B-type supergiants (Crowther et al. 2006; Markova \& Puls 2008), although it provides a constraint for a detailed analysis of the equation of motion when the effect of a rotating star is considered.

Inclusion of rotation in the models brought remarkable progress in the development of the theory of stellar winds. Besides the classical $m$-CAK solution (Friend \& Abbott 1986; Pauldrach et al. 1986), hereafter called the fast solution, Curé (2004) discovered the so-called $\Omega$-slow solution for radiationdriven winds in stars rotating at speeds close to their critical

\footnotetext{
${ }^{3}$ Member of the Carrera del Investigador Científico, CONICET, Argentina.
}

(breakup) velocities, $V_{\text {crit }}$, i.e., at $\Omega \gtrsim 0.7-0.8$, with $\Omega=V_{\text {rot }} /$ $V_{\text {crit }}$, where $V_{\text {rot }}$ is the rotation velocity at the star's equator. As compared with the fast solution, the $\Omega$-slow solution leads to a dense and slow wind, characterized by a lower terminal velocity. It is worth mentioning that both regimes predict almost the same mass-loss rate, but the fast solution tends to deliver lower mass-loss rates.

Curé \& Rial (2004) explored the topology of the CAK solution (point star approximation) assuming a wind frozen in ionization ( $\delta=0$, where $\delta$ is the ionization-related line-force parameter; Abbott 1982) and concluded that the effect of the rotation is to shift the location of the singular point downstream. Thus, the $C A K$ solution is the only physical solution. Then, these authors analyzed for this model all the solutions for $\delta$ values ranging from 0.0 to 0.2 (typical values given by the standard $m-C A K$ theory) and found a bifurcation in the solution topology, with the concurrence of two critical points: one is the known standard $C A K$ critical point, and the other is a topological attractor located closer to the star surface.

Later on, Curé et al. (2011) showed the existence of another type of slow hydrodynamical solution that results when the parameter $\delta$ is assumed to be higher than the values provided by the standard $m$-CAK formalism (Abbott 1982; Puls et al. 2000). High values of $\delta$, even larger than $\delta=1 / 3$, which corresponds to a medium with neutral hydrogen as a trace element (Puls et al. 2000), are expected in strong ionization gradients. This hydrodynamical solution, called the $\delta$-slow solution, possesses low terminal velocities, similar to those predicted by the $\Omega$-slow solution, but without the requirement of a high-speed rotator. The $\delta$-slow solution exists even for a nonrotating case. This solution fits quite well the requirements of the anomalous correlation between $V_{\infty} / V_{\text {esc }}$ and $V_{\text {esc }}$ and the wind momentum-luminosity relationship observed in A supergiants (Curé et al. 2011). However, these 
authors did not explore completely the domain of the $\delta$-slow solution to derive the parameter space for which $\delta$ produces a switch between fast and slow outflowing regimes. Therefore, many important topics concerning the behavior of the $\delta$-slow solution still remain unknown: (1) How is the $\delta$-slow solution linked to the stellar rotation? (2) What are the values of $\delta$ that lead to a switch between fast and slow outflowing regimes? (3) How do the slow solutions correlate with other fundamental parameters of the star, such as effective temperature, stellar mass, radius, metallicity, etc.? (4) Do the slow solutions represent the wind structure of B supergiants?

This work is devoted to answering some of the above questions, mainly when both the rotation $(\Omega)$ and different wind ionization structures $(\delta)$ are present. To this purpose, we perform a numerical analysis of the domains where the fast, $\Omega$ slow, and $\delta$-slow solutions might exist. We explore the resulting solutions considering an extended range of values for $\delta$, in combination with a fine grid of rotation rates represented by $\Omega$. In addition, we set the main parameters of our test models for B supergiants, for which the standard theory gives unsatisfactory estimates of the global parameters of their winds.

In Section 2 we briefly present the basic hydrodynamic equations for rotating radiation-driven winds and define the grid of parameters for the models of this research. Section 3 shows the resulting values of mass-loss and terminal velocity for each solution of the hydrodynamic equations and also gives a description of the domains for the fast, $\Omega$-slow, and $\delta$-slow solutions. A discussion about the different wind regimes, a discussion of the presence of a gap separating fast from slow stationary solutions, and a brief comparison with observations are given in Section 4. Conclusions and work in progress are addressed in Section 5.

\section{THE HYDRODYNAMICAL WIND MODEL}

As was described in Curé (2004) and Curé et al. (2011), the wind velocity profile is obtained by solving the radial component of the momentum equation at the equatorial plane,

$$
\begin{aligned}
v \frac{d v}{d r}= & -\frac{1}{\rho} \frac{d p}{d r}-\frac{G M_{*}(1-\Gamma)}{r^{2}}+\frac{v_{\phi}^{2}(r)}{r} \\
& +g \operatorname{line}\left(\rho, \frac{d v}{d r}, n_{\mathrm{E}}\right),
\end{aligned}
$$

together with the mass conservation or continuity equation,

$$
F_{\mathrm{m}}=r^{2} \rho v .
$$

where $F_{\mathrm{m}}$ is the mass flux.

Expressing $\rho$ as a function of $v$ from Equation (2) and replacing it in Equation (1), we obtain the equation of motion, hereafter EoM. The numerical solutions of the EoM give the mass-loss rate, ${ }^{4} \dot{M}=4 \pi F_{\mathrm{m}}$, the radial velocity of the fluid, $v$ $(r)$, and the mass density distribution of the wind, $\rho(r)$, as a function of the radial coordinate. The first term on the righthand side of Equation (1) represents the force due to the gas pressure $p(r)$. The second term corresponds to the gravity force, where $G$ is the universal gravitational constant, $M_{*}$ the stellar mass, and $\Gamma$ the correction due to the acceleration of the continuum radiation. The third term is the centrifugal force, and

\footnotetext{
The expected mass-loss rate if we assume a spherical star.
}

Table 1

Main Parameters for the Basic Models of B Supergiant Stars

\begin{tabular}{lccccc}
\hline \hline Name & $\begin{array}{c}T_{\text {eff }} \\
(\mathrm{K})\end{array}$ & $\begin{array}{c}\log g \\
(\mathrm{cgs})\end{array}$ & $\begin{array}{c}R_{*} \\
\left(R_{\odot}\right)\end{array}$ & $k$ & $\alpha$ \\
\hline T13 & 13000 & 1.73 & 68 & 0.30 & 0.5 \\
T15 & 15000 & 2.11 & 52 & 0.32 & 0.5 \\
T17 & 17000 & 2.24 & 56 & 0.34 & 0.5 \\
T19 & 19000 & 2.50 & 40 & 0.32 & 0.5 \\
T21 & 21000 & 2.67 & 35 & 0.32 & 0.5 \\
T23 & 23000 & 2.72 & 35 & 0.34 & 0.53 \\
T25 & 25000 & 2.90 & 35 & 0.34 & 0.55 \\
\hline
\end{tabular}

the last one is the radiative acceleration $g^{\text {line }}$ due to momentum absorption in line transitions.

We adopt the $m$-CAK standard parameterization for the line acceleration,

$$
g^{\text {line }}=\frac{C}{r^{2}} f_{\mathrm{D}}(r, v, d v / d r)\left(r^{2} v \frac{d v}{d r}\right)^{\alpha}\left(\frac{n_{\mathrm{E}}(r)}{W(r)}\right)^{\delta}
$$

which is expressed in terms of three line-force parameters $(k, \alpha$, and $\delta$ ). The function $f_{\mathrm{D}}$ is the finite disk correction factor, $W(r)$ is the dilution factor, and $\boldsymbol{n}_{\mathrm{E}}(\boldsymbol{r})$ is the electron number density. The line-force parameter $k$ is included in the constant $C$ (eigenvalue), which depends on the mass-loss rate in the form

$$
C=\Gamma G M k\left(\frac{4 \pi}{\sigma_{\mathrm{E}} v_{\mathrm{th}} \dot{M}}\right)^{\alpha},
$$

where $\sigma_{\mathrm{E}}$ is the Thomson scattering constant and $v_{\mathrm{th}}$ is the thermal velocity of the fluid.

The $C A K$ line-force parameters $k, \alpha$, and $\delta$ are closely related to physical properties of the wind. For instance, $k$ and $\alpha$ respectively describe the statistical dependence of lines on frequency position and line strength, while $\delta$ is related to the change in the ionization of the wind.

Alternative formulations of the radiation force (Müller \& Vink 2008) can also be expressed in terms of the $m$-CAK lineforce parameters (Araya et al. 2014).

In order to solve the above equations, we make use of the code Hydwind, which is extensively described by Curé (2004). The main parameters adopted for modeling the stars are summarized in Table 1. Each entry of the table is considered a "basic model." The first column is the short name of the basic model, and the following columns are effective temperature, $T_{\text {eff }}$, surface gravity, $\log g$, stellar radius $R_{*}$ in solar units, and the values adopted for the line-force parameters, $k$ and $\alpha$. Most of the values adopted in this table are based on the line-force parameters ( $\alpha$ and $k$ ) reported by Pauldrach et al. (1986) and Abbott (1982), while the fundamental stellar parameters are basically similar to the ones published by Searle et al. (2008). All the models were computed using solar abundances. For each of the "basic models" we look for the hydrodynamic solution considering a grid of rotation rates ranging from $\Omega=0.0$ to 0.9 , with intervals of 0.1 , and $\delta$ values ranging from 0.0 to 0.4 , in steps of 0.01 . We find that the precision of this grid is good enough to satisfy our purposes on defining the boundaries of each solution domain.

In this work, we solve the $m$-CAK EoM considering the boundary condition $\tau_{*}=2 / 3$ at the stellar surface, for the 
Table 2

Values of $v_{\infty}$ and $\dot{M}$ for Computed Models with $T_{\text {eff }}=19,000 \mathrm{~K}$ (T19)

\begin{tabular}{|c|c|c|c|c|c|c|c|c|c|c|c|c|c|c|c|c|c|c|c|c|}
\hline \multirow{3}{*}{$\delta$} & \multicolumn{20}{|c|}{$\Omega$} \\
\hline & \multicolumn{2}{|c|}{0.0} & \multicolumn{2}{|c|}{0.1} & \multicolumn{2}{|c|}{0.2} & \multicolumn{2}{|c|}{0.3} & \multicolumn{2}{|c|}{0.4} & \multicolumn{2}{|c|}{0.5} & \multicolumn{2}{|c|}{0.6} & \multicolumn{2}{|c|}{0.7} & \multicolumn{2}{|c|}{0.8} & \multicolumn{2}{|c|}{0.9} \\
\hline & $v_{\infty}$ & $\dot{M}$ & $v_{\infty}$ & $\dot{M}$ & $v_{\infty}$ & $\dot{M}$ & $v_{\infty}$ & $\dot{M}$ & $v_{\infty}$ & $\dot{M}$ & $v_{\infty}$ & $\dot{M}$ & $v_{\infty}$ & $\dot{M}$ & $v_{\infty}$ & $\dot{M}$ & $v_{\infty}$ & $\dot{M}$ & $v_{\infty}$ & $\dot{M}$ \\
\hline 0.00 & 724.8 & 1.206 & 719.5 & 1.219 & 703.4 & 1.261 & 676.1 & 1.337 & 636.3 & 1.458 & 582.0 & 1.645 & 509.6 & 1.939 & 407.5 & 2.426 & 217.8 & 2.395 & 198.9 & 2.433 \\
\hline 0.01 & 704.1 & 1.224 & 699.0 & 1.237 & 683.3 & 1.281 & 656.5 & 1.360 & 617.5 & 1.485 & 564.4 & 1.680 & 492.9 & 1.989 & 389.6 & 2.498 & 216.1 & 2.418 & 197.3 & 2.463 \\
\hline 0.02 & 684.3 & 1.242 & 679.2 & 1.256 & 663.9 & 1.301 & 637.7 & 1.383 & 599.5 & 1.514 & 547.3 & 1.718 & 476.6 & 2.041 & 234.8 & 2.401 & 214.5 & 2.444 & 195.7 & 2.497 \\
\hline 0.03 & 665.1 & 1.260 & 660.2 & 1.275 & 645.2 & 1.322 & 619.5 & 1.408 & 582.0 & 1.544 & 530.6 & 1.758 & 460.7 & 2.096 & 233.1 & 2.422 & 212.8 & 2.472 & 194.1 & 2.532 \\
\hline 0.04 & 646.7 & 1.280 & 641.8 & 1.296 & 627.1 & 1.344 & 601.9 & 1.433 & 565.1 & 1.576 & 514.5 & 1.799 & 445.0 & 2.154 & 231.3 & 2.443 & 211.2 & 2.502 & 192.6 & 2.572 \\
\hline 0.05 & 628.9 & 1.300 & 624.1 & 1.317 & 609.6 & 1.368 & $\ldots$ & $\ldots$ & 548.7 & 1.610 & 498.8 & 1.843 & 429.5 & 2.216 & 229.6 & 2.468 & 209.6 & 2.536 & 191.0 & 2.615 \\
\hline 0.06 & 611.6 & 1.322 & 607.0 & 1.339 & 592.8 & 1.392 & $\cdots$ & $\cdots$ & 532.9 & 1.645 & 483.4 & 1.890 & 414.0 & 2.282 & 227.9 & 2.495 & 207.9 & 2.571 & 189.5 & 2.661 \\
\hline 0.07 & 595.0 & 1.344 & 590.4 & 1.362 & 576.5 & 1.417 & 552.5 & 1.519 & 517.4 & 1.682 & 468.5 & 1.940 & 398.3 & 2.352 & 226.1 & 2.524 & 206.3 & 2.611 & 187.9 & 2.712 \\
\hline 0.08 & 578.9 & 1.367 & 574.3 & 1.386 & 560.6 & 1.444 & 537.1 & 1.550 & 502.4 & 1.722 & 453.7 & 1.993 & $\cdots$ & $\cdots$ & 224.4 & 2.557 & 204.7 & 2.654 & 186.4 & 2.767 \\
\hline 0.09 & 563.2 & 1.392 & 558.8 & 1.411 & 545.3 & 1.472 & 522.0 & 1.584 & 487.7 & 1.764 & 439.3 & 2.049 & 244.3 & 2.497 & 222.6 & 2.592 & 203.1 & 2.701 & 184.9 & 2.827 \\
\hline 0.10 & 548.1 & 1.418 & 543.7 & 1.438 & 530.4 & 1.502 & 507.4 & 1.619 & 473.4 & 1.809 & 424.9 & 2.109 & 242.1 & 2.526 & 220.9 & 2.631 & 201.5 & 2.753 & 183.4 & 2.894 \\
\hline 0.11 & 533.3 & 1.445 & 529.0 & 1.467 & 515.9 & 1.534 & 493.2 & 1.656 & 459.4 & 1.856 & 410.6 & 2.173 & 239.9 & 2.557 & 219.1 & 2.673 & 199.9 & 2.809 & $\ldots$ & $\ldots$ \\
\hline 0.12 & 519.0 & 1.475 & 514.7 & 1.497 & 501.7 & 1.567 & 479.2 & 1.696 & 445.6 & 1.907 & 396.2 & 2.242 & 237.9 & 2.592 & 217.4 & 2.720 & 198.3 & 2.871 & $\ldots$ & $\ldots$ \\
\hline 0.13 & 505.1 & 1.506 & 500.8 & 1.529 & 488.0 & 1.603 & 465.6 & 1.739 & 432.0 & 1.962 & $\ldots$ & $\ldots$ & 235.9 & 2.630 & 215.7 & 2.772 & 196.7 & 2.939 & $\ldots$ & $\ldots$ \\
\hline 0.14 & 491.5 & 1.539 & 487.3 & 1.563 & 474.5 & 1.641 & 452.3 & 1.785 & 418.5 & 2.021 & $\ldots$ & $\ldots$ & 233.9 & 2.672 & 214.0 & 2.829 & 195.2 & 3.014 & $\ldots$ & $\ldots$ \\
\hline 0.15 & 478.2 & 1.574 & 474.0 & 1.600 & 461.3 & 1.682 & 439.1 & 1.834 & 405.1 & 2.084 & $\ldots$ & $\ldots$ & 231.9 & 2.719 & 212.3 & 2.893 & 193.6 & 3.098 & $\ldots$ & $\ldots$ \\
\hline 0.16 & 465.2 & 1.612 & 461.0 & 1.640 & 448.4 & 1.727 & 426.2 & 1.888 & 391.4 & 2.153 & $\ldots$ & $\ldots$ & 229.9 & 2.772 & 210.6 & 2.963 & 192.1 & 3.191 & $\ldots$ & $\ldots$ \\
\hline 0.17 & 452.4 & 1.653 & 448.3 & 1.682 & 435.6 & 1.774 & 413.3 & 1.945 & $\ldots$ & $\ldots$ & 249.0 & 2.648 & 228.0 & 2.830 & 208.9 & 3.041 & 190.5 & 3.293 & $\ldots$ & $\ldots$ \\
\hline 0.18 & 439.9 & 1.697 & 435.8 & 1.728 & 423.1 & 1.826 & 400.4 & 2.008 & $\cdots$ & $\ldots$ & 246.3 & 2.698 & 226.1 & 2.896 & 207.2 & 3.129 & 189.0 & 3.408 & $\ldots$ & $\cdots$ \\
\hline 0.19 & 427.5 & 1.745 & 423.4 & 1.778 & 410.6 & 1.882 & 387.3 & 2.076 & $\ldots$ & $\ldots$ & 243.7 & 2.753 & 224.1 & 2.969 & 205.5 & 3.226 & 187.5 & 3.536 & $\ldots$ & $\ldots$ \\
\hline 0.20 & 415.3 & 1.797 & 411.1 & 1.832 & 398.1 & 1.943 & $\ldots$ & $\ldots$ & $\cdots$ & $\ldots$ & 241.3 & 2.815 & 222.2 & 3.051 & 203.8 & 3.335 & 186.0 & 3.680 & $\ldots$ & $\ldots$ \\
\hline 0.21 & 403.1 & 1.854 & 398.9 & 1.891 & 385.4 & 2.010 & $\cdots$ & $\cdots$ & $\cdots$ & $\cdots$ & 238.9 & 2.885 & 220.3 & 3.143 & 202.2 & 3.458 & 184.6 & 3.843 & $\cdots$ & $\cdots$ \\
\hline 0.22 & 390.9 & 1.916 & 386.5 & 1.957 & $\ldots$ & $\ldots$ & $\cdots$ & $\cdots$ & 257.0 & 2.716 & 236.6 & 2.963 & 218.4 & 3.247 & 200.5 & 3.597 & 183.2 & 4.027 & $\cdots$ & $\cdots$ \\
\hline 0.23 & 378.3 & 1.986 & 373.4 & 2.029 & $\ldots$ & $\ldots$ & $\ldots$ & $\ldots$ & 253.1 & 2.792 & 234.4 & 3.053 & 216.6 & 3.366 & 198.9 & 3.755 & 181.8 & 4.236 & $\ldots$ & $\ldots$ \\
\hline 0.24 & $\ldots$ & $\ldots$ & $\ldots$ & $\ldots$ & $\ldots$ & $\ldots$ & $\ldots$ & $\ldots$ & 249.9 & 2.873 & 232.2 & 3.154 & 214.7 & 3.501 & 197.3 & 3.936 & 180.4 & 4.474 & $\ldots$ & $\ldots$ \\
\hline 0.25 & $\ldots$ & $\ldots$ & $\ldots$ & $\ldots$ & $\ldots$ & $\ldots$ & $\ldots$ & $\ldots$ & 247.0 & 2.964 & 230.0 & 3.270 & 212.9 & 3.655 & 195.8 & 4.143 & 179.2 & 4.748 & $\ldots$ & $\ldots$ \\
\hline 0.26 & $\ldots$ & $\ldots$ & $\ldots$ & $\ldots$ & $\ldots$ & $\ldots$ & 261.4 & 2.789 & 244.3 & 3.067 & 227.9 & 3.403 & 211.1 & 3.833 & 194.2 & 4.382 & 178.1 & 5.061 & $\ldots$ & $\ldots$ \\
\hline 0.27 & $\cdots$ & $\cdots$ & $\cdots$ & $\cdots$ & $\cdots$ & $\cdots$ & 257.2 & 2.894 & 241.7 & 3.186 & 225.8 & 3.558 & 209.3 & 4.039 & 192.7 & 4.660 & 177.2 & 5.420 & $\cdots$ & $\cdots$ \\
\hline 0.28 & $\ldots$ & $\ldots$ & $\ldots$ & $\ldots$ & $\ldots$ & $\ldots$ & 253.7 & 3.008 & 239.2 & 3.323 & 223.7 & 3.737 & 207.6 & 4.280 & 191.3 & 4.984 & 176.5 & 5.830 & $\ldots$ & $\ldots$ \\
\hline 0.29 & $\ldots$ & $\ldots$ & $\ldots$ & $\ldots$ & 262.9 & 2.882 & 250.5 & 3.138 & 236.7 & 3.484 & 221.7 & 3.947 & 205.9 & 4.562 & 190.0 & 5.364 & 176.0 & 6.298 & $\ldots$ & $\ldots$ \\
\hline 0.30 & 270.8 & 2.775 & 267.2 & 2.842 & 258.8 & 3.017 & 247.5 & 3.289 & 234.3 & 3.672 & 219.7 & 4.196 & 204.2 & 4.898 & 188.9 & 5.808 & 176.0 & 6.831 & $\ldots$ & $\ldots$ \\
\hline 0.31 & 265.3 & 2.926 & 262.6 & 2.989 & 255.2 & 3.169 & 244.7 & 3.467 & 232.0 & 3.897 & 217.7 & 4.492 & 202.7 & 5.298 & 188.1 & 6.326 & 176.4 & 7.434 & $\ldots$ & $\ldots$ \\
\hline 0.32 & 261.1 & 3.087 & 258.7 & 3.153 & 252.0 & 3.348 & 242.0 & 3.679 & 229.7 & 4.165 & 215.9 & 4.849 & 201.3 & 5.777 & 187.7 & 6.929 & 177.2 & 8.111 & $\cdots$ & $\ldots$ \\
\hline 0.33 & 257.4 & 3.273 & 255.2 & 3.345 & 248.9 & 3.561 & 239.4 & 3.934 & 227.5 & 4.491 & 214.1 & 5.282 & 200.1 & 6.353 & 187.7 & 7.619 & 178.6 & 8.666 & $\ldots$ & $\ldots$ \\
\hline 0.34 & 254.0 & 3.496 & 251.9 & 3.576 & 246.0 & 3.820 & 236.9 & 4.246 & 225.4 & 4.892 & 212.5 & 5.813 & 199.3 & 7.039 & 188.4 & 8.402 & 180.6 & 9.703 & $\ldots$ & $\ldots$ \\
\hline 0.35 & 250.8 & 3.767 & 248.9 & 3.858 & 243.2 & 4.138 & 234.5 & 4.632 & 223.5 & 5.388 & 211.1 & 6.468 & 199.2 & 7.843 & 189.8 & 9.280 & 183.2 & 10.62 & $\ldots$ & $\ldots$ \\
\hline 0.36 & 247.8 & 4.105 & 246.0 & 4.210 & 240.6 & 4.537 & 232.2 & 5.118 & 221.7 & 6.013 & 210.1 & 7.269 & 199.7 & 8.764 & 191.9 & 10.26 & $\ldots$ & $\ldots$ & $\ldots$ & $\ldots$ \\
\hline 0.37 & 245.0 & 4.533 & 243.3 & 4.657 & 238.1 & 5.045 & 230.2 & 5.738 & 220.2 & 6.801 & 209.9 & 8.227 & 201.2 & 9.801 & 194.8 & 11.33 & $\cdots$ & $\cdots$ & $\cdots$ & $\cdots$ \\
\hline 0.38 & 242.4 & 5.088 & 240.8 & 5.237 & 235.9 & 5.704 & 228.4 & 6.538 & 219.3 & 7.783 & 210.6 & 9.332 & 203.6 & 10.95 & 198.5 & 12.51 & $\cdots$ & $\cdots$ & $\cdots$ & $\cdots$ \\
\hline 0.39 & 240.1 & 5.820 & 238.5 & 6.002 & 234.0 & 6.569 & 227.2 & 7.563 & 219.4 & 8.961 & 212.5 & 10.57 & 207.0 & 12.22 & 203.0 & 13.80 & $\ldots$ & $\ldots$ & $\ldots$ & $\ldots$ \\
\hline 0.40 & 238.2 & 6.799 & 236.8 & 7.020 & 232.7 & 7.700 & 226.9 & 8.834 & 220.9 & 10.30 & 215.6 & 11.93 & 211.4 & 13.60 & 208.3 & 15.18 & $\ldots$ & $\ldots$ & $\ldots$ & $\ldots$ \\
\hline
\end{tabular}

The units are $\mathrm{km} \mathrm{s}^{-1}$ and $10^{-6} M_{\odot} \mathrm{yr}^{-1}$, respectively. 

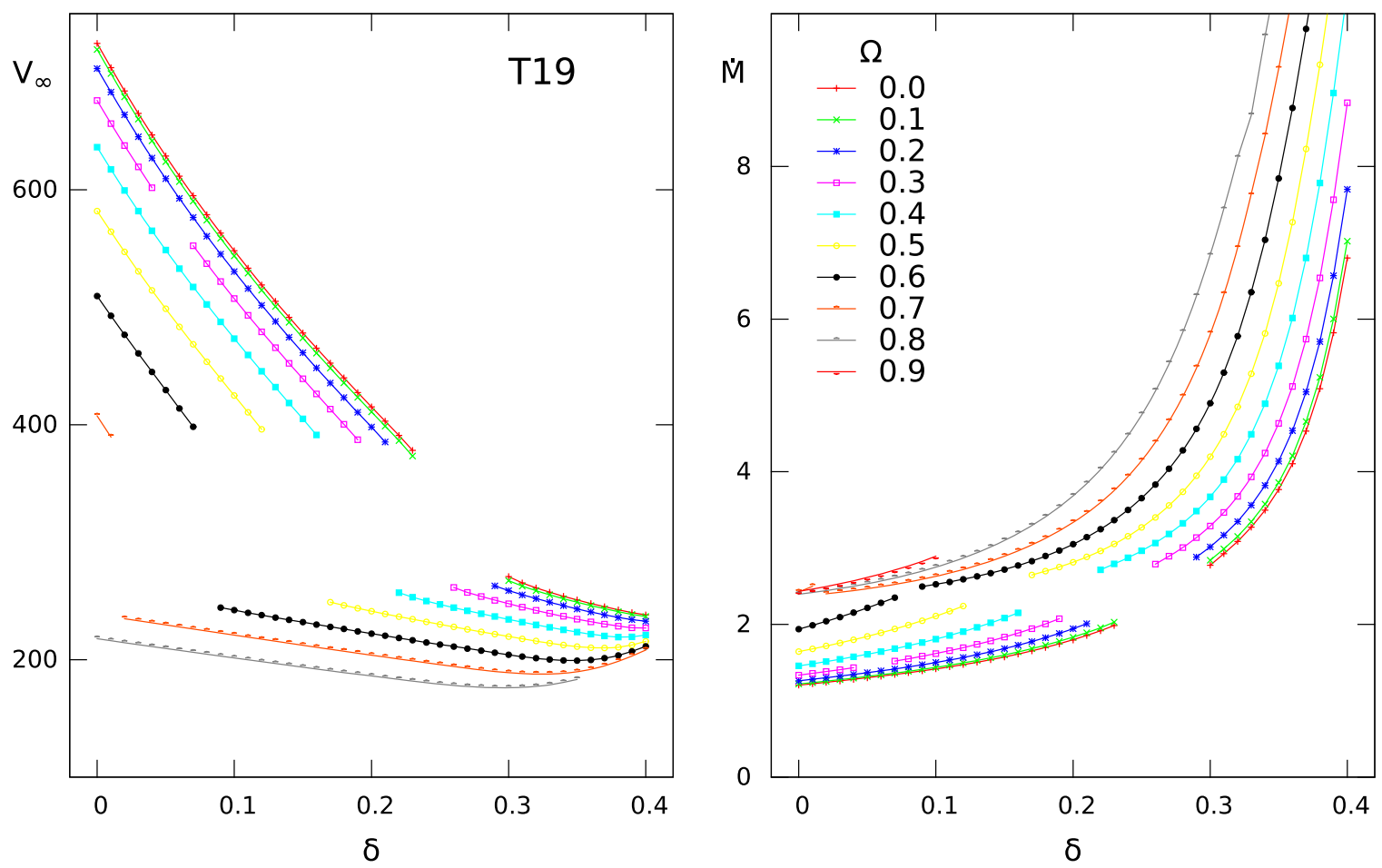

Figure 1. Left: terminal velocities in $\mathrm{km} \mathrm{s}^{-1}$ as a function of the parameter $\delta$ and the rotation rate (in color lines with dots) for model T19. Right: mass-loss rates in units of $10^{-6} M_{\odot} \mathrm{yr}^{-1}$ as a function of $\delta$ and $\Omega$. Note the gap that separates fast from slow solutions.

monochromatic optical depth $\tau_{*}$ defined as

$$
\tau_{*}=\int_{R_{*}}^{\infty} \sigma_{\mathrm{E}} \rho(r) d r
$$

instead of fixing the value of the mass density at the same layer.

\section{RESULTS}

After solving the EoM for the "basic models" listed in Table 1, we found that the radiation-driven wind solution for stars with low $T_{\text {eff }}$ (lower than 23,000 K) shows a different behavior than the resulting one from stars with high $T_{\text {eff. }}$. In this section we present the results of the calculations and summarize the main wind properties.

\subsection{Flow Regimes for Stars with Low $T_{\text {eff }}$}

\subsubsection{Distribution of the Solution Domains}

In order to give a global overview of the nature of the hydrodynamic solutions for the winds of mid- and late B-type supergiants, it is first convenient to select from Table 1 one "basic model." To this purpose we choose the "basic model" T19. Then we report on the differences found with the remaining "basic models."

A complete set of the global wind parameters $v_{\infty}$ (terminal velocity) and $\dot{M}$, related to model T19, are obtained for various $\Omega$ and $\delta$ values (see Table 2). For some combinations of $\Omega$ and $\delta$ parameters, no stationary solution is found by HYDwIND, and a blank space is left in the corresponding places of the table. As the HYDwIND code needs a trial velocity law for starting and boosting the convergence rate, we apply a trial-and-error approach, looking for the best well-behaved converged solution. For each box that remains empty in Table 2, we have attempted about 6000 runs of test parameters with no result. Therefore, we are quite confident that there are no simple stationary solutions for some combination of $\Omega$ and $\delta$ parameters.

The distribution of the tabulated values of $v_{\infty}$ and $\dot{M}$ has a straightforward interpretation (see Table 2). The solution obtained for the basic model T19, considering low values of $\delta$, is consistent with the one expected for a fast classical radiationdriven wind solution, with the exception of the results obtained for $\Omega \gtrsim 0.8$ that switch to a slow flow regime (Curé 2004; Madura et al. 2007). The behavior of all solutions can be seen in Figure 1, where the terminal velocities (left panel) and massloss rates (right panel) are plotted against $\delta$. Each curve, represented by different colors (electronic version) and different symbols, is the wind solution for a given $\Omega$. For the studied range of parameters it often results that the lower $\delta$, the higher $v_{\infty}$ and the lower $\dot{M}$. The top left branches of each sequence of curves (Figure 1, left panel) correspond to the fast solution. Their respective mass-loss rates are represented by the bottom left branches seen in Figure 1 (right panel).

At some value of $\delta$, let us say $\delta_{\min }$, which depends strongly on $\Omega$, the fast solution vanishes. The lack of a stationary physical solution continues for a limited interval of consecutive values of $\delta$, higher than $\delta_{\min }$, which originate a gap. Then for values of $\delta$ higher than those that encompass the upper limit of the gap, $\delta \geqslant \delta_{\max }$, a physical stationary wind solution is attained again, but now the found solution ( $\delta$-slow solution) corresponds to a flow regime with lower terminal velocity and higher mass-loss rate than the fast solution.

Both the location and the width of the gap depend on $\Omega$. If $\Omega$ is lower than $\sim 0.8$, the gap takes place at higher values of $\delta$. As the rotation rate increases, the starting $\delta$-value, $\delta_{\min }$, diminishes and the gap becomes thinner until it vanishes completely at $\Omega \simeq 0.8$. For larger values of $\Omega$ no fast solution can be found for any given value of $\delta$. As an example, we can observe that in 

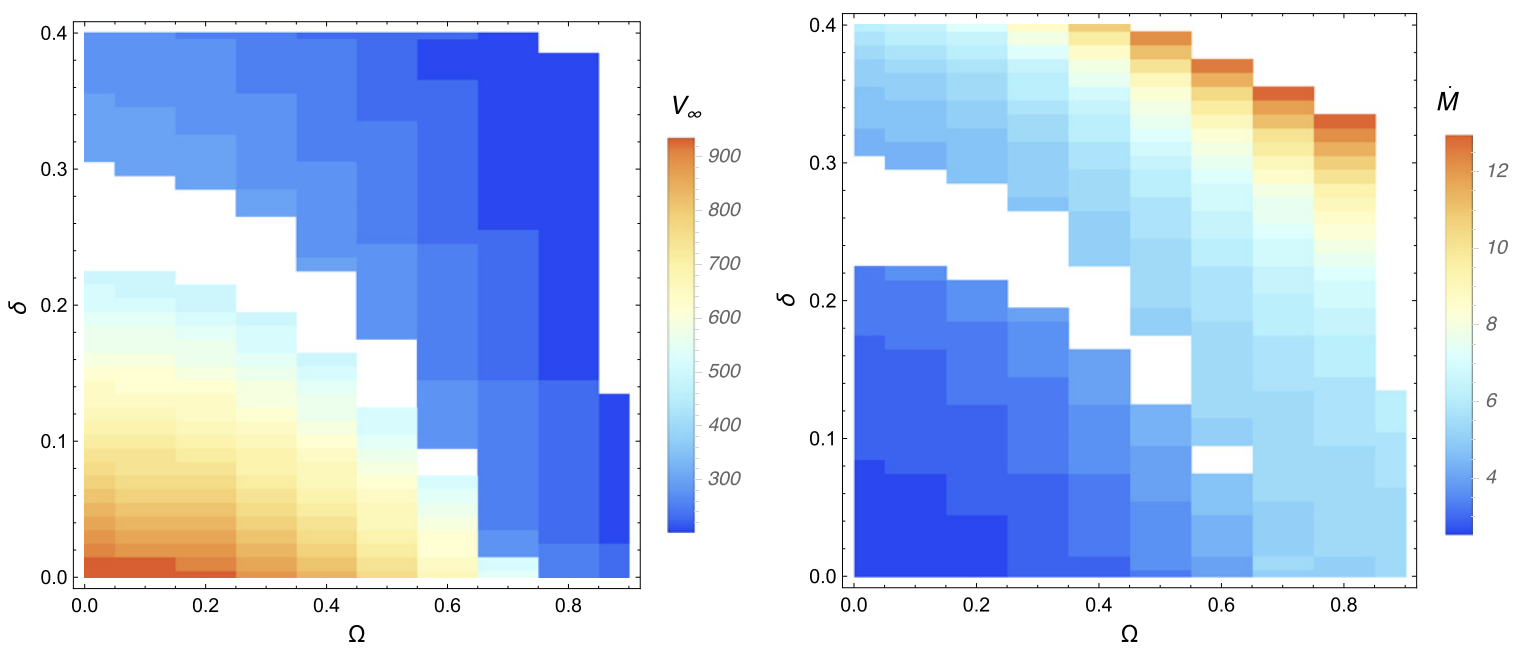

Figure 2. Color maps representing the behavior of terminal velocities (left panel) and mass-loss rates in units of $10^{-6} M_{\odot} \mathrm{yr}^{-1}$ (right panel) corresponding to the "basic model" T19, as a function of the parameter $\delta$. In the first plot, the region below the gap (blank region) corresponds to the fast solution, while the above bluish zone contains the slow solution. In the second plot, the region below the gap corresponds to the fast regime, while the upper region corresponds to the slow one.

the "basic nonrotating model" T19 $(\Omega=0.0)$ the width of the gap $\left(\Delta \delta=\delta_{\max }-\delta_{\min }\right)$, in a $\delta$ scale, is 0.07 , while for $\Omega=0.6$ it shrinks to 0.02 . At $\Omega=0.7$ the gap is even smaller (0.01) and is located between $\delta=0.1$ and 0.2 (see Table 2). Finally, for $\Omega \gtrsim 0.8, \Delta \delta=0$. The location and the width of the gaps, which represent a discontinuity in the solutions, are illustrated in Figure 1.

After the gap, for $\delta \geqslant \delta_{\max }$, we find the domain of the slow wind solution where the variation of the terminal velocity is small, in contrast with the behavior of $\dot{M}$, which strongly enhances as $\delta$ increases. This is shown in the right branches of Figure 1 (left panel for the terminal velocity and right panel for the mass-loss rate). Figure 2 shows color maps for the different solution domains, terminal velocities, and mass-loss rates corresponding to the "basic model" T19. It is noticeable that there is a gap between fast and slow solutions (blank region) and that there is no sharp boundary between $\delta$-slow and $\Omega$-slow regimes.

The rotation speed contributes to enlarge the extent of the slow solution domain. As the rotation rate raises, the gap shifts toward lower values of $\delta$ and the slow solution domain advances over the fast solution domain.

In the case of rapidly rotating stars $(\Omega \geqslant 0.8)$, the solution is of the type of the $\Omega$-slow solution, since the centrifugal acceleration term reduces the effective gravity and moves the critical point leeward the location of the CAK sonic point. As a consequence, a lower velocity gradient is established in the subsonic region.

On the other hand, in the domain of slow rotators, the term of the radiation force, expressed through the optical depth factor $t^{-\alpha}$, dominates the momentum equation. Particularly, the presence of ionization gradients (expressed by a factor of the form $\left(n_{\mathrm{E}} / W\right)^{\delta}$ in Equation (1)) introduces an additional density dependence, and its overall effect can be roughly accounted for by substituting $\alpha$ for $\alpha_{\text {eff }}=\alpha-\delta$. The higher the $\delta$, the lower the $\alpha_{\text {eff }}$, with the consequent increment of $\dot{M}$ and decrement of the wind speed. This kind of solution is of the type of the $\delta$-slow regime found by Curé et al. (2011).

The inverse variation of $\dot{M}$ and $v_{\infty}$ can be understood by realizing that the product $\dot{M} v_{\infty}$ is the momentum loss of the wind, which, for slowly rotating radiation-driven winds, scales with the momentum loss of the radiation $L / c$. In Figure 3 we

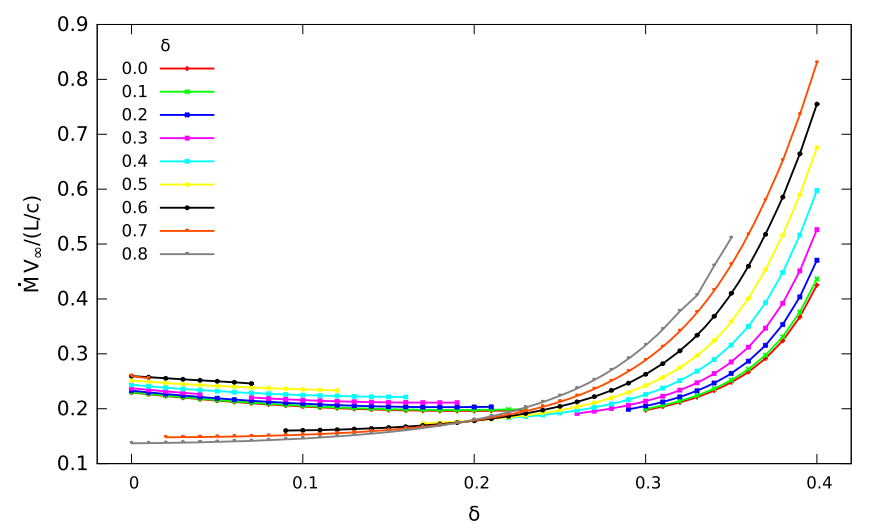

Figure 3. Ratio between wind and radiation momentum losses vs. $\delta$ for model T19. The momentum transfer becames more efficient within the slow solution domain.

plot this behavior for model T19. We can observe there that within the fast model domain $\dot{M} v_{\infty}$ is almost constant, in agreement with what it is expected. This dependence holds even for high rotators with low $\delta$ values. However, in the slow $\delta$ domain (high $\delta$ ), the momentum loss enhances strongly as $\delta$ increases. This means that the momentum transfer is more efficient owing to the fact that the abundance of line-driving ions decreases more steeply with distance than in a wind with small values of $\delta$.

\subsubsection{Comparison with Other Models of Low $T_{\text {eff }}$}

All the models with $T_{\text {eff }} \leqslant 21,000 \mathrm{~K}$ show analogous behaviors akin to the already-described model T19 (see Figures 4 and 5). This means that they supply a fast solution when $\delta \leqslant \delta_{\min }$ and a slow solution for $\delta \geqslant \delta_{\max }$. Both sets of solutions are separated by a gap. Fast rotators $(\Omega \geqslant 0.8)$ do not deliver fast solutions at any value of $\delta$, as Curé (2004) demonstrated.

\subsubsection{Remarks on the Gap}

Figure 6 (left panel) plots the average of $\delta$ values at both sides of the gap as a function of $\Omega$, providing a rough reference of the gap location in a $\delta$ scale. In all the models it is clear that 

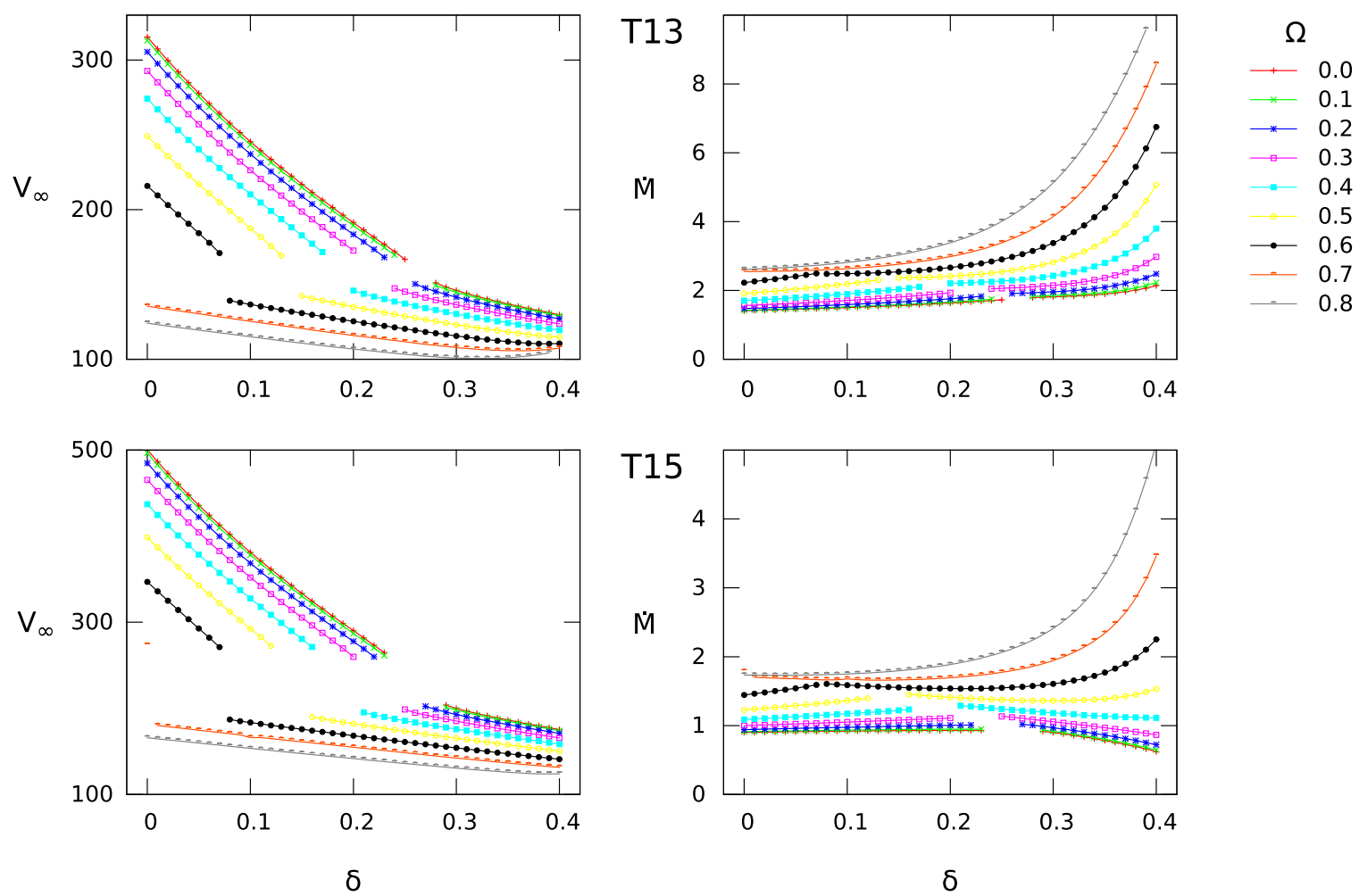

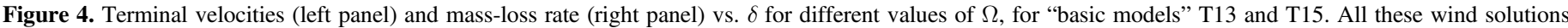
exhibit the same domain distribution, with a gap clearly dividing the fast and the slow regimes.
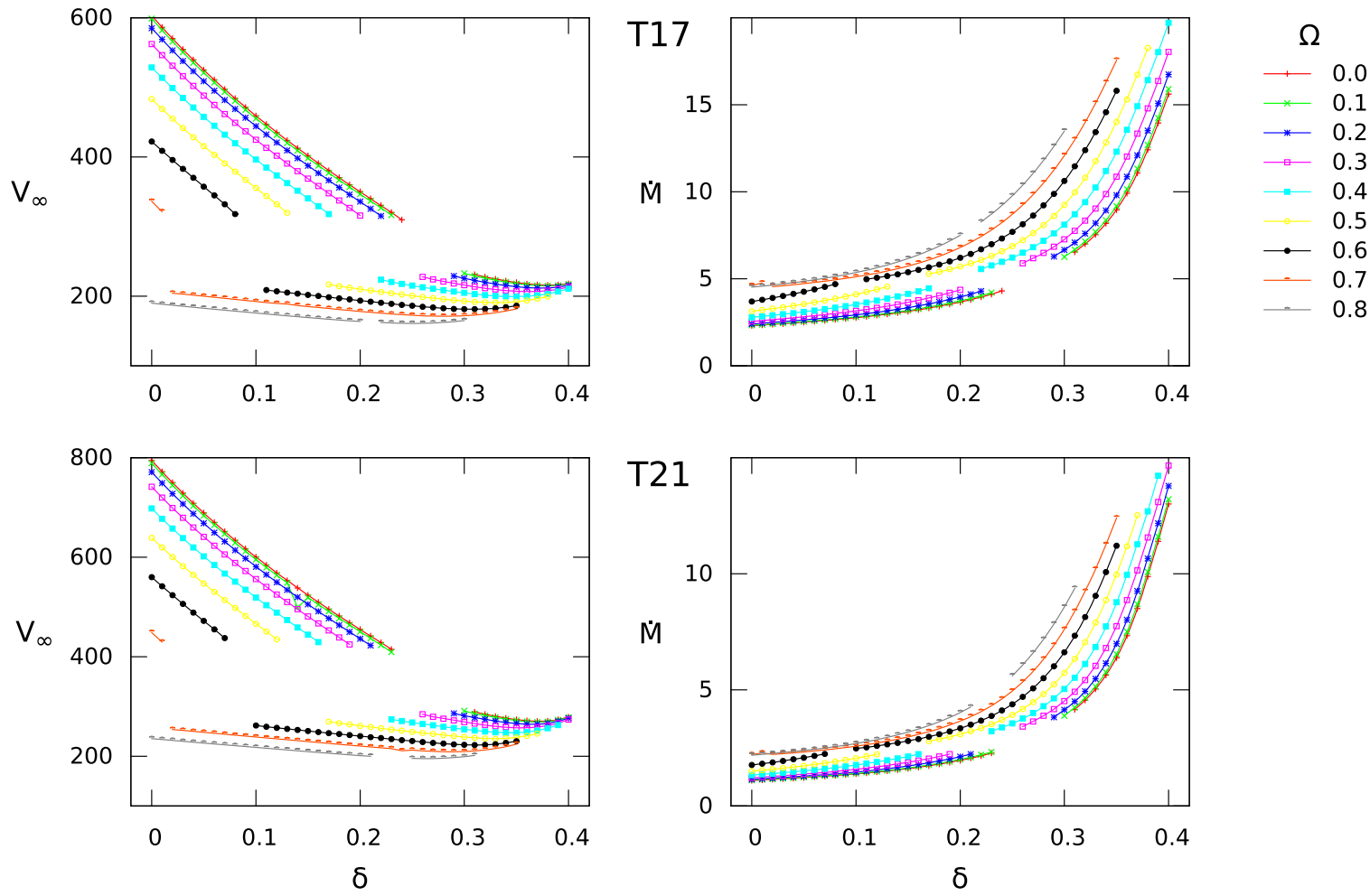

Figure 5. Same as Figure 4 but for "basic models" T17 and T21.

the gap shifts toward lower values of $\delta$ as $\Omega$ increases. In addition, this figure shows that the gap is centered at almost the same values of $\delta$ even for different values of $T_{\text {eff. }}$ On the other hand, Figure 6 (right panel) illustrates the variation of the gap width ( $\Delta \delta=\delta_{\max }-\delta_{\min }$ in a $\delta$ scale), which diminishes as $\Omega$ increases. In this figure, the sequence of basic models with $T_{\text {eff }}$ values lower than or equal to $21,000 \mathrm{~K}$ displays a similar behavior. 

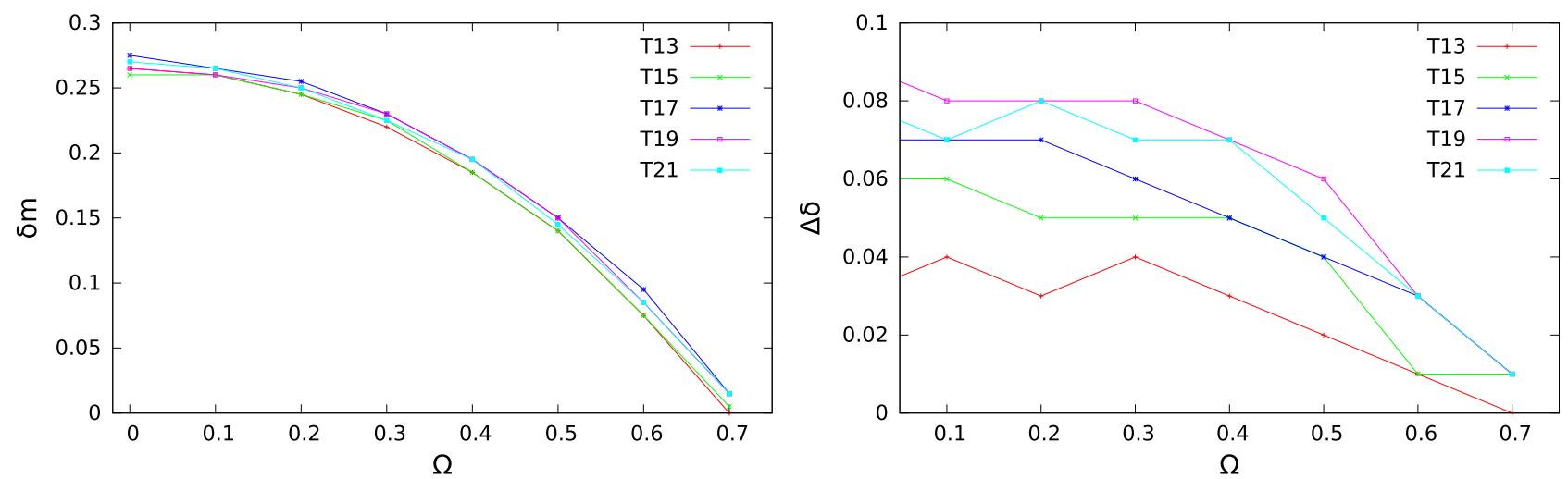

Figure 6. Left: mean value of the gap borders $\delta_{m}=\left(\delta_{\min }+\delta_{\max }\right) / 2$ (i.e., the location of the gap in $\delta$ scale) vs. $\Omega$ for models with $T_{\text {eff }} \leqslant 21,000 \mathrm{~K}$. The gap is located closely at almost the same value of $\delta$. Right: width of the gap in a $\delta$ scale vs. $\Omega$ for the "basic models" with $T_{\text {eff }} \leqslant 21,000 \mathrm{~K}$. The higher the rotation rate, the narrower the gap.
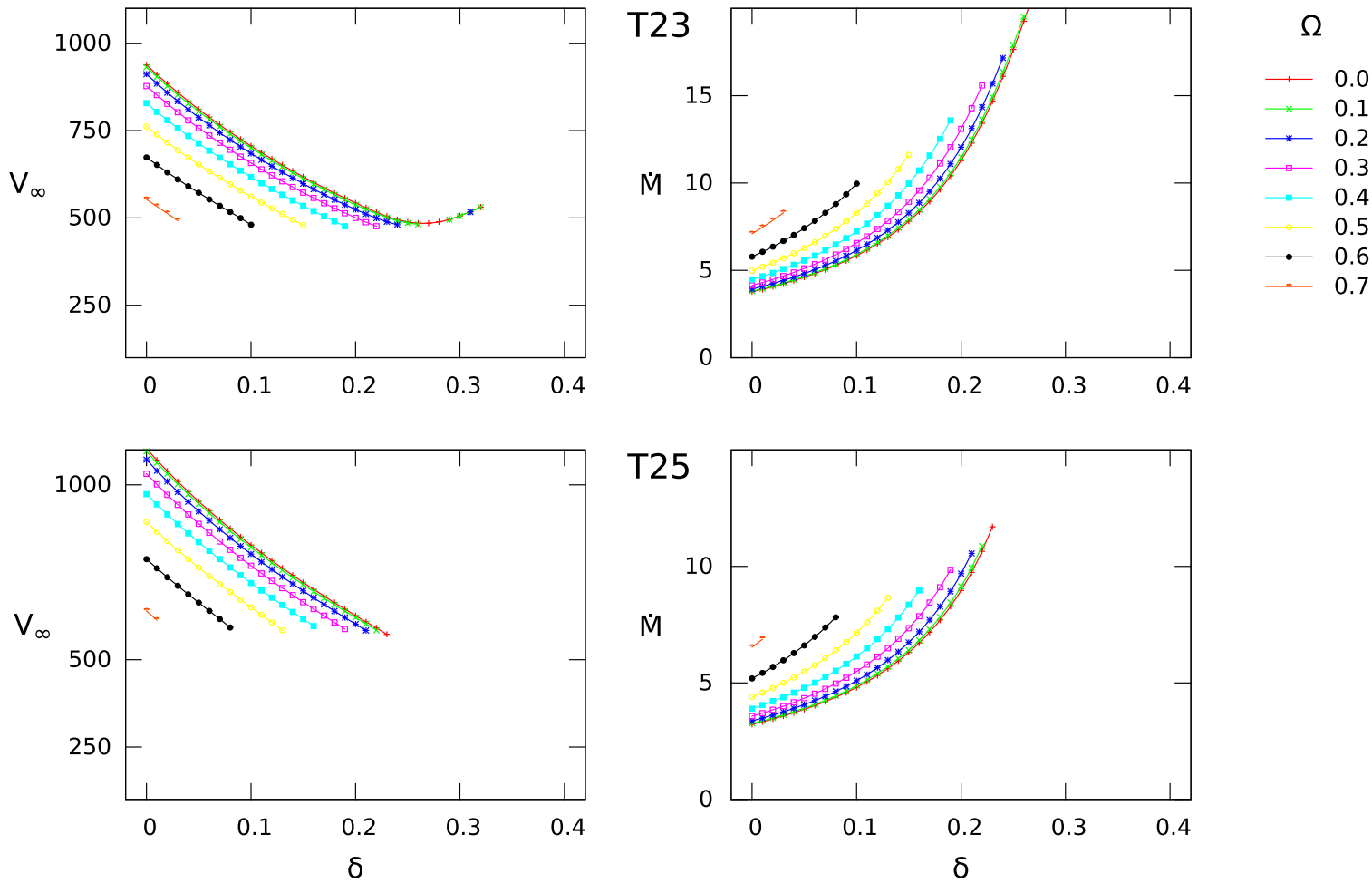

Figure 7. Same as Figure 4 but for "basic models" T23 and T25. The slow solution is not present for the explored range of parameters.

\subsection{Flow Regimes for Stars with High $T_{\text {eff }}$}

\subsubsection{Distribution of the Solution Domains}

For the tested models with effective temperatures larger than $21,000 \mathrm{~K}$, which are representative of early B-type supergiants, the EoM does not achieve a converged solution for any value of $\delta>\delta_{\min }$ and any given $\Omega$ parameter. Therefore, in the range of the parameters tabulated in Table 1, the solution domain is restricted only to the fast wind regime. Figure 7 depicts this result for models T23 and T25, where the solution remains for $\delta$ values (from 0.0 to around 0.25 ) lower than the typical $\delta_{\text {min }}$ found in the basic models with low $T_{\text {eff. }}$ This range of $\delta$ values, which yields a fast solution, is in accordance with the values predicted by the classical $m$ - $C A K$ model.

However, it is worth mentioning that the existence of the $\Omega$ slow solution has been reported by Curé (2004) and Madura et al. (2007) for effective temperatures greater than or equal to
25,000 K, but with a different set of $\alpha$ and $k$ line force parameter. We performed some tests for the basic model T25 using a lower value of $k$, and we were able to recover a small set of slow solutions. Therefore, in a forthcoming paper we plan to extend the grid of $\alpha$ and $k$ parameters over the allowed available parameter space of each model (R. O. J. Venero et al. 2016, in preparation). The reliability of these values will be discussed by contrasting with observations.

\section{DISCUSSION}

\subsection{Mathematical Properties of the Wind Solutions}

From the analysis of the behavior of the 1D hydrodynamical solution presented in Section 3, it is clear that the line-force parameter $\delta$, which accounts for changes of ionization throughout the wind, is one of the responsible variables (together with $\Omega$ ) for setting either a fast or a slow regime. 

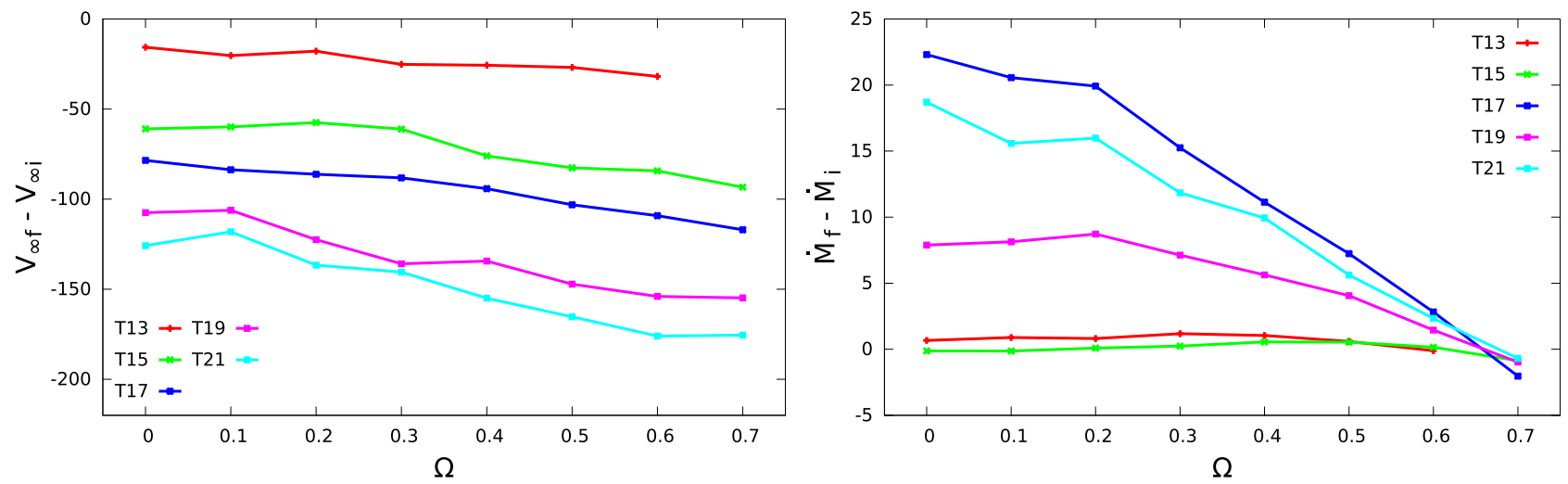

Figure 8. Left: jump in $V_{\infty}$ (in $\mathrm{km} \mathrm{s}^{-1}$ ) between both boundaries of the gaps vs. $\Omega$, for the "basic models" referred to in the legend. Right: same as the left panel but for $\dot{M}$ (in units of $10^{-6} M_{\odot} \mathrm{yr}^{-1}$ ).

Table 3

Known Stationary Solutions for Radiation-driven Winds

\begin{tabular}{|c|c|c|c|c|c|}
\hline Wind Regime & Solution Type & Properties & $\Omega$ Range & $\delta$ Range & $V_{\infty} / V_{\text {esc }}$ \\
\hline fast & m-CAK & $\begin{array}{l}\text { large acceleration } \\
\text { low rotation } \\
\text { constant ionization }\end{array}$ & $<0.8$ & $\lesssim 0.25$ & $\sim 0.9-2.3$ \\
\hline \multirow{2}{*}{ slow } & $\Omega$-slow & $\begin{array}{l}\text { slow acceleration } \\
\text { high rotation }\end{array}$ & $\geqslant 0.8$ & $\lesssim 0.25$ & $\sim 0.5-0.7$ \\
\hline & $\delta$-slow & $\begin{array}{l}\text { slow acceleration } \\
\text { ionization gradients }\end{array}$ & $<0.8$ & $>\delta_{\max }$ & $\lesssim 0.9$ \\
\hline
\end{tabular}

Note. The tabulated values are rough reference values that correspond to the models computed in this work. They could depend on other line-force parameters such as $k$ and $\alpha$. Parameter $\delta_{\max }$ is the boundary at higher $\delta$ of the gap and depends on both $\Omega$ and $T_{\text {eff }}$.

Values of $\delta$ lower than $\delta_{\min }$ give rise to the standard $m$-CAK solution (a fast wind regime) in agreement with most of the published rotating hydrodynamic models. On the other hand, high values of $\delta$, greater than $\delta_{\max }$, are reflected in the $\delta$-slow solution.

In the slow solution domain, two regions should be distinguished where $\delta$ and $\Omega$ values dominate one over the other. These are the $\delta$-slow regime, for high ionization gradients along winds (high $\delta$ ) and low values of $\Omega$, and the $\Omega$-slow regime, for fast rotators $(\Omega \geqslant 0.8)$ and a low $\delta$. We note that there is a soft or no transition between both regions, leading to a single common $\Omega \delta$-slow regime, as can be seen from Table 2.

Fast and slow solution domains are separated by a gap at an interval delimited by $\delta_{\min }<\delta<\delta_{\max }$, where a nonstationary solution has been found with Hydwind. For fast rotators, this gap is placed at lower values of $\delta$. Hence, rotation modulates the location and the width of the gap. There is also a dependence of the parameters of the gap on $T_{\text {eff }}$ and the lineforce parameters $\alpha$ and $k$.

The existence of the gap has been overlooked in previous studies since all of them restricted their analysis to the $\delta$ values given by the standard $m$-CAK model. Nevertheless, Curé \& Rial (2004), in their analysis of the topology of the $C A K$ solution, found a bifurcation when non-null $\Omega$ and $\delta$ values were considered simultaneously. They also found a forbidden region where no critical point could be located (Curé \& Rial 2004, see Figure 6). In our opinion, the gap that we are reporting here could correspond to the analytical prolongation of the forbidden region found by Curé \& Rial (2004).
From now on, we restrict our analysis to stars with effective temperatures in the interval between 13,000 and $21,000 \mathrm{~K}$, where the gap structure is present. The gap separates regions of stationary solutions with different terminal velocities and massloss rates. The differences between the terminal velocities at both sides of the gap are small for the models with low effective temperature. The difference in velocity increases toward higher values of $T_{\text {eff }}$ and $\Omega$. This result is displayed in Figure 8 (left panel). Instead, the difference of mass-loss rate diminishes as $\Omega$ increases (Figure 8 , right panel). The relation for $\dot{M}$ is steeper in the early B subtypes. In this panel, the sequence of curves might not be continuous owing to the choice of the $k$ parameter, on which the mass loss depends strongly.

The main mathematical properties of the stationary solutions for rotating radiation-driven winds that have been discussed in the present paper are sketched in Table 3. The fast regime corresponds to a high accelerated wind solution with $V_{\infty} / V_{\text {esc }}$ ratios greater than 0.9 , while the slow regime results when a low $d v / d r$ is established and the $V_{\infty} / V_{\text {esc }}$ ratios are lower than 0.9 .

One question still unanswered is about the possible structure of the wind for the parameter space of the gap where no stationary solution was found with the code HYDwind. We could wonder whether the wind settles down to another stationary behavior or whether it turns into a chaotic timedependent regime. As the gap links a region dominated by a fast regime with another with a slow outflowing flow, we can glimpse that the wind regime would be unstable and turn from fast to slow wind regimes and vice versa. 


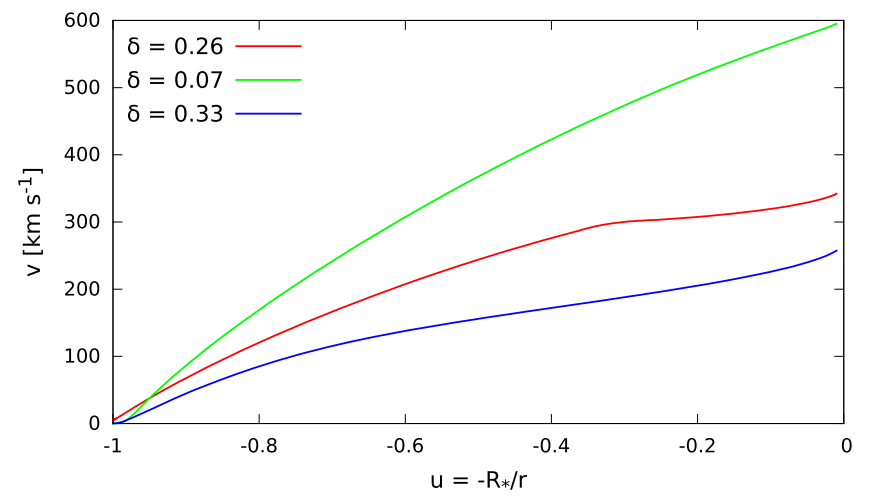

Figure 9. Velocity fields computed with the ZEUS-3D code for a set of parameters corresponding to the fast domain (green line), the slow domain (blue line), and the gap (red line). Note that the solution corresponding to the gap shows a kink or plateau structure.

To have a first glance about the wind structure inside the gap, we perform a preliminary computation of the wind hydrodynamics using the time-dependent code ZEUS-3D (Clarke 1996, 2010) under the same assumptions used with HYDwIND (i.e., 1D computation and radial forces). We used three different sets of parameters representative of the gap region and of the fast and slow solution domains. It is worth mentioning that the code ZEUS-3D reproduces similar results to the ones obtained previously with HYDwIND, but, in addition, it provides a solution inside the gap. Figure 9 shows the corresponding velocity fields obtained with the basic model T19: a fast solution with $\delta=0.07$ (green line), a $\delta$-slow solution with $\delta=0.33$ (blue line), and a solution for the conditions inside the gap $(\delta=0.26$, red line), presenting a sudden deceleration. The solution found inside the gap seems to be stationary and displays a kink/plateau velocity field structure.

Solutions with a kink structure have been discussed by Cranmer \& Owocki (1996) to explain the formation of discrete absorption components (DACs) in O-type stars. Further studies on the stability and properties of this solution are worth being studied.

\subsection{Physical Properties and Applications of the Models}

In order to evaluate whether these models are capable of representing the main features of a B supergiant wind, we must first determine the constraints of their major assumptions. The adopted 1D geometry is one of the basic hypotheses to discuss from the beginning. Therefore, unless we model a fast rotator $(\Omega \gtrsim 0.9)$ where the geometry of the system is strongly axisymmetrical, we might accept that the wind structure is nearly spherical and that the obtained radial component of the velocity field is a representative solution at any latitude. In fact, most of the B supergiants satisfy this condition since the rotational velocity of early $B$ supergiants is around $100 \mathrm{~km} \mathrm{~s}^{-1}$; this value decreases toward late spectral types, being about 30 or $40 \mathrm{~km} \mathrm{~s}^{-1}$ for A supergiants (Kudritzki et al. 1999). As expected, 2D and 3D models enable us to explore many characteristics of the winds that we cannot do with our simple 1D model, e.g., the influence of radial forces on the formation of disk-like structure (Bjorkman \& Cassinelli 1993) or the effects of nonradial forces inhibiting it (Owocki et al. 1996), and the formation of prolate non-LTE winds when gravity darkening is considered (Petrenz \& Puls 2000).
A second hypothesis to discuss is the use of the finite disk spherical correction factor. As we are dealing with slow rotators, we can neglect the oblate distorsion of the shape of the star due to stellar rotation. However, this effect would be very important in the case of fast rotators (i.e., B[e] supergiants). As a consequence of the distorsion of the stellar surface, the star's oblateness will contribute to accelerating a polar wind and originate slower and denser winds toward the equatorial plane. Preliminary calculations on the effects of the oblate correction factor were discussed by Araya et al. (2011), who found that the topology of the hydrodynamical differential equation is certainly modified and other critical points may exist. Therefore, new hydrodynamical solutions are expected.

The third hypothesis involved in our models is related to the assumption of a constant thermal velocity throughout the wind. In principle, we expect that it has negligible effects on the solutions when solving the momentum equation, since the contribution of thermal pressure gradients corresponding to changes of a few tens of thousands of kelvins would be of a second order, with respect to the line-radiation acceleration. However, the assumption of a constant temperature is a weakness in our models since a nonconstant temperature would change the wind ionization structure. In this work, these ionization gradients are taken into account indirectly by increasing the value of $\delta$ (which is assumed to be constant along the wind), and in this way it defines the wind regime and stability.

The role of high-temperature gradients would also be remarkable in very fast rotators (e.g., B[e] stars), where a decrease in temperature from polar to equatorial regions is expected. This effect would change the ionization structure of the flow with latitude, leading to different wind regimes that favor the formation of outflowing disk or kink structures. Models based on this assumption were discussed by Zickgraf et al. (1985), Lamers \& Pauldrach (1991), and Curé et al. (2005). In the last two works, the authors also analyzed the effects of the bi-stability temperature jump as a mechanism to produce a switch in the wind regime at a given latitude. The main issue of these models is that they are restricted to critical rotators with $T_{\text {eff }}$ close to the temperature of the bi-stability jump (21,000 K; Lamers et al. 1995). Instead, the advantage of the $\delta$-slow solution is that it holds for a wider effective temperature range $\left(T_{\text {eff }}<21,000 \mathrm{~K}\right)$ and has no restriction on the rotation velocity of the star. This suggests that gradients of temperature would favor disk formation in noncritical rotators or allow the development of instabilities in wind, by switching the wind conditions between regimes.

Another neglected effect in our models is the contribution of meridional flows between polar and equatorial zones. These flows, expected in very fast rotators, would increase the density at the equatorial plane, making the disk structure more relevant. This problem, which is beyond the scope of the present work, cannot be described by our simple model, and a 2D treatment should be mandatory.

In spite of the simple assumptions of a spherical and isothermal wind, our models enable us to describe quite well the global features of the wind, such as mass-loss rates and terminal velocities of moderately and slowly rotating $B$ supergiants. This agreement relies on the fact that most of the B supergiants are slow rotators and that their thermal pressure gradients are not so important. 


\subsection{Transitions between Wind Regimes}

In many circumstances, the ionization conditions along the wind could change, producing a transit between two regimes limited by the particular conditions around a gap. This is very likely to occur along the evolution of a B supergiant, as $T_{\text {eff }}$ and ionization conditions of the fluid slowly change. Hence, we would expect switches between fast and slow outflowing regimes, or vice versa. These mechanisms could contribute to the understanding of phases of variable mass-loss rate along the evolutionary stages.

Also, the fact that the $\delta$-slow solution seems not to be present in stars with $T_{\text {eff }}>21,000 \mathrm{~K}$ restricts the possibility of a switch between fast and slow regimes at such high temperatures. That would provide an alternative physical explanation of the empirical bi-stability jump observed around $21,000 \mathrm{~K}$ in $\mathrm{B}$ supergiants (Lamers et al. 1995). These possibilities should be explored in more detail and supported by observations.

In a short-term scale, another long-standing problem of early-type stars is the presence of blueshifted narrow absorption features (or DACs) superimposed on the wide absorption of the P Cygni profiles (Morton 1976; Gathier et al. 1981). These DACs are evident in a wide variety of objects, namely, W-R, $\mathrm{O}$, early binaries, B supergiants, Be stars, and Central Stars of Planetary Nebulae (CSPN) (Prinja 1990). Many researchers have pointed out that DACs are features related to optical depth enhancements, originated by the presence of plateaus in the velocity fields (Lamers et al. 1982). The wind solution obtained with ZEUS-3D using conditions characteristic of a gap region (Figure 9) resembles this scenario, with a kink/plateau on the velocity fields. A temporal sequence of models for the transition of a wind across a gap could explain why DACs evolve accelerating through line profiles toward higher velocity as they diminish its widths.

The evolution of DACs was also reported in some binary systems during half of the orbital period (Arias et al. 2008). To describe this phenomenon, we propose that the presence of a hot companion overheats local regions on the star surface, originating a bright spot, which can produce a local change in the ionization and trigger a switch in the wind regime. This switch should be phased with the orbital motion, giving rise to line profile variations along the orbital period. This way, the presence of the $\delta$-slow solution and the switch mechanism may support the Co-rotating Interaction Regions model (CIRs; Cranmer \& Owocki 1996) proposed to explain the DACs. It would be interesting to explore whether such a scheme is suitable to describe the behavior of the observed features.

\subsection{Wind Properties along the Spectral Sequence of $B$ Supergiants}

As is shown in Figure 6 (left panel), the required values of $\delta$ that might enable a switch between two different wind regimes are almost the same for the entire considered $T_{\text {eff }}$ range. However, the switch is more probable for stars with low $T_{\text {eff }}$ and high $\Omega$, because of the thin width of the gap (Figure 6, right panel).

It is generally accepted that most of the $\mathrm{O}$ and early $\mathrm{B}$ supergiants can be modeled properly with a $\beta$ velocity law with $\beta<1$. However, as is mentioned in Section 1 , A and late $\mathrm{B}$ supergiants exhibit notorious departures from these values, since often $\beta \geqslant 2$. The difference in the behavior between both groups (early B- and late B/A-type supergiants) is clearly seen

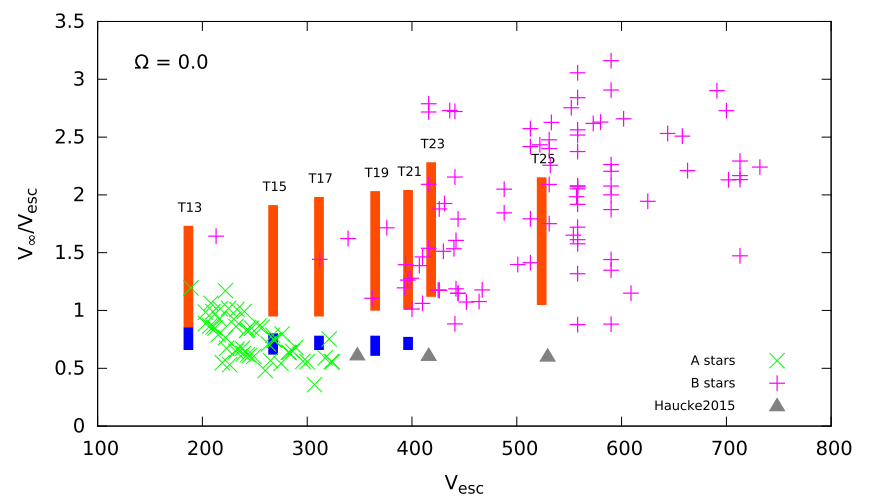

Figure 10. $V_{\infty} / V_{\text {esc }}$ vs. $V_{\text {esc }}$ relationship comparing models with observations. Vertical red bars correspond to the full range of the fast solution for the labeled basic models and computed for the related values of $\delta$. Blue vertical bars represent the range of $V_{\infty} / V_{\text {esc }}$ values for the slow regime. The blue bars could extend even more toward low values of $V_{\infty} / V_{\text {esc }}$ than plotted here, because we impose a limit value for $\delta(0.4)$.

in the empirical $V_{\infty} / V_{\text {esc }}$ versus $V_{\text {esc }}$ relationship shown in Figure 10 (symbols represent observations from Prinja et al. 1990; Lamers et al. 1995; Achmad et al. 1997; Verdugo et al. 1998), which shows two different slopes. This figure also shows a comparison between the observations and the results of our models. Vertical red bars, plotted for different basic models, represent the range of $V_{\infty} / V_{\text {esc }}$ values corresponding to the fast solution with different $\delta$ values and $\Omega=0$. This fast solution fits quite well the terminal velocities measured in early and mid-B supergiants. Instead, the small vertical blue bars that correspond to the slow solution regime match properly early A supergiants and possibly some B late-type stars (Figure 10, upward-pointing triangles). Values of $V_{\infty}$ and line-force parameters for the late B supergiants, which seem to be an extension of the A supergiants, were derived by fitting the observed line profiles with those computed with the slow solution (Haucke et al. 2015).

Further remarks on the solutions obtained with models for

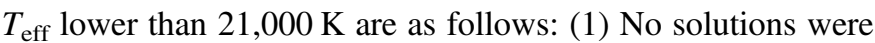
found for $\Omega \geqslant 0.9$ for basic models T13, T15, and, T17, which correspond to the latest subtype stars. Instead, a few models with low values of $\delta$ and very high values of $\Omega$ were found for T19 and T21 basic models. (2) $\dot{M}$ decreases when $\delta$ increases, i.e., see the slow solution for basic models T15 $\left(T_{\text {eff }}=15,000 \mathrm{~K}\right)$ with a low $\Omega$. While the dependence of $v_{\infty}$ on $\Omega$ and $\delta$ seems to follow the same trend, in agreement with the behavior described for the rest of the basic models, the slow solution obtained for T15 presents a relationship between $\dot{M}$ and $\delta$ with a negative slope. Hydrodynamical models for fast O-type rotators also showed decreases in the mass-loss rate toward the equator in response to the computed line acceleration (Müller \& Vink 2014). From our results we support that this peculiar behavior could be attained not only for dependence of $\dot{M}$ on $\delta$ but also for the ratio $T_{\text {eff }} / \log g$ for a given set of $k$ and $\alpha$ line-force parameters. Extended research on this dependence for an oblate star is currently under development.

\section{CONCLUSIONS}

In this work we perform a numerical study of the 1D hydrodynamical solution for massive spherical rotating stars to obtain the flow behavior in the $\delta-\Omega$ domain, using the condition of $\tau_{*}=2 / 3$ on the surface of the star. To this 
purpose, we consider stellar fundamental parameters of typical $\mathrm{B}$ supergiants. The radiation force is modeled within the frame of the $m$ - $C A K$ theory, with the exception that the range of $\delta$ was spread out to take into account different ionization structures. We also analyze the behavior of the solution with $\Omega$ (the rotation rate). We find well-defined domains that can lead to either fast or slow outflowing regimes.

At high $T_{\text {eff }}$, hotter than $21,000 \mathrm{~K}$, only the fast standard $m$ $C A K$ solutions seem to be present among the selected range of parameters. For $T_{\text {eff }} \leqslant 21,000 \mathrm{~K}$ and a given rotation speed, low values of $\delta\left(\delta<\delta_{\min }\right)$ yield a fast outflowing regime with an almost constant wind-momentum loss, while high values of $\delta\left(\delta>\delta_{\max }\right)$ lead to a slow expanding wind with a higher momentum removal efficiency. No wind solution was found with HYDwind for values of $\delta$ inside the interval $\delta_{\min }<\delta<\delta_{\max }$. However, a time-dependent hydrodynamical calculation yields a stationary solution inside the gap that displays a kink/plateau velocity field structure.

The location of this gap shifts toward the lowest values of $\delta$ and becomes narrower as the rotation rate $\Omega$ increases. In the domain of the fastest rotators, only the slow solutions remain and there is no evident difference between the behavior of the $\delta$-slow solution close to the critical rotation speed and the known behavior of the $\Omega$-slow solution reported by Curé (2004). Therefore, we conclude that both solutions belong to the same family of solutions, and we propose to call them the $\Omega \delta$-slow solutions.

We also show that the terminal velocities observed in early and mid-B supergiants fit the results of fast outflowing winds while those of late B and, mainly, early A supergiants match the results for slow stationary outflowing wind regimes.

We propose that it would be possible to have a switch between fast and slow regimes, originated by changes in the ionization conditions, leading to wind variations. This effect could be more important in fast rotators because the variation in $\delta$ needed to jump the gap is considerably small.

On the other hand, the fact that the $\delta$-slow solution seems not to be present in stars with $T_{\text {eff }}>21,000 \mathrm{~K}$ restricts the possibility of a switch between fast and slow regimes at such high temperatures. That would provide an alternative physical explanation of the empirical bi-stability jump observed around $21,000 \mathrm{~K}$ in B supergiants (Lamers et al. 1995). In addition, as a consecuence of this switch, a star has to go through (or remain inside) the gap; therefore, it should show the evolution of DACs.

In a forthcoming paper, which is currently under development, we will present extended research on the relevance of the remaining line-force parameters, $\alpha$ and $k$, and evaluate their effects on the solutions together with $\Omega$ and $\delta$. We also plan to replace the condition of $\tau_{*}$ by a fixed value of the photospheric density. In addition, we plan to compute the emergent spectral line profiles and discuss the applications of the current known hydrodynamic solutions to the outflowing winds observed in many B supergiants. The nature of the wind regime inside a gap and its related effects are worth exploring in detail. All these new results would allow us to revisit important features of the winds of massive stars, like the evolution of DACS and the wind momentum-luminosity relationship.

We thank our anonymous referee for many helpful comments and suggestions. R.O.J.V. and L.C. acknowledge financial support from the Agencia de Promoción Científica y Tecnológica (Préstamo BID PICT 2011/0885), CONICET (PIP 0177), and the Universidad Nacional de La Plata (Programa de Incentivos G11/137, and PPID/G003), Argentina. M.C. thanks the support from FONDECYT (project 1130173) and the Centro de Astrofísica de Valparaíso Chile. I. A. is grateful for the support from Gemini-CONICYT 32120033 (Grants for the Astronomy Graduate Program of the Universidad de Valparaíso) and Fondo Institucional de Becas FIB-UV.

\section{REFERENCES}

Abbott, D. C. 1982, ApJ, 259, 282

Achmad, L., Lamers, H. J. G. L. M., \& Pasquini, L. 1997, A\&A, 320, 196 Araya, I., Curé, M., \& Cidale, L. S. 2014, ApJ, 795, 81

Araya, I., Curé, M., Granada, A., \& Cidale, L. S 2011, in IAU Symp. 272, Active OB Stars: Structure, Evolution, Mass Loss and Critical Limits, ed. C. Neiner et al. (Cambridge: Cambridge Univ. Press), 83

Arias, M. L., Sahade, J., \& Barbá, R. H. 2008, RMxAC, 33, 118

Bjorkman, J. E., \& Cassinelli, J. P. 1993, ApJ, 409, 429

Castor, J. I., Abbott, D. C., \& Klein, R. I. 1975, ApJ, 195, 157

Clarke, D. A. 1996, ApJ, 457, 291

Clarke, D. A. 2010, ApJS, 187, 119

Cranmer, S. R., \& Owocki, S. P. 1996, ApJ, 462, 469

Crowther, P. A., Lennon, D. J., \& Walborn, N. R. 2006, A\&A, 446, 279

Curé, M. 2004, ApJ, 614, 929

Curé, M., Cidale, L., \& Granada, A. 2011, ApJ, 737, 18

Curé, M., \& Rial, D. F. 2004, A\&A, 428, 545

Curé, M., Rial, D. F., \& Cidale, L. 2005, A\&A, 437, 929

Friend, D. B., \& Abbott, D. C. 1986, ApJ, 311, 701

Gathier, R., Lamers, H. J. G. L. M., \& Snow, T. P. 1981, ApJ, 247, 173

Haucke, M., Araya, I., Arcos, C., et al. 2015, in IAU Symp. 307, New Windows on Massive Stars Asteroseismology, Interferometry, and Spectropolarimetry, ed. G. Meynet et al. (Cambridge: Cambridge Univ. Press), 104

Kudritzki, R. P., Puls, J., Lennon, D. J., et al. 1999, A\&A, 350, 970

Lamers, H. J. G., \& Pauldrach, A. W. A. 1991, A\&A, 244, L5

Lamers, H. J. G. L. M., Gathier, R., \& Snow, T. P., Jr. 1982, ApJ, 258, 186

Lamers, H. J. G. L. M., \& Rogerson, J. B., Jr. 1978, A\&A, 66, 417

Lamers, H. J. G. L. M., Snow, T. P., \& Lindholm, D. M. 1995, ApJ, 455, 269

Madura, T. I., Owocki, S. P., \& Feldmeier, A. 2007, ApJ, 660, 687

Markova, N., \& Puls, J. 2008, A\&A, 478, 823

Morton, D. C. 1976, ApJ, 203, 386

Müller, P. E., \& Vink, J. S. 2008, A\&A, 492, 493

Müller, P. E., \& Vink, J. S. 2014, A\&A, 564, A57

Owocki, S. P., Cranmer, S. R., \& Gayley, K. G. 1996, ApJL, 472, L115

Pauldrach, A., Puls, J., \& Kudritzki, R. P. 1986, A\&A, 164, 86

Petrenz, P., \& Puls, J. 2000, A\&A, 358, 956

Prinja, R. K. 1990, MNRAS, 246, 392

Prinja, R. K., Barlow, M. J., \& Howarth, I. D. 1990, ApJ, 361, 607

Puls, J., Springmann, U., \& Lennon, M. 2000, A\&AS, 141, 23

Searle, S. C., Prinja, R. K., Massa, D., \& Ryans, R. 2008, A\&A, 481, 777

Verdugo, E., Talavera, A., \& Gómez de Castro, A. I. 1998, Ap\&SS, 263, 263

Verdugo, E., Talavera, A., \& Gómez de Castro, A. I. 1999, A\&AS, 137, 351

Zickgraf, F.-J., Wolf, B., Stahl, O., Leitherer, C., \& Klare, G. 1985, A\&A, 143, 421 